\title{
Article
}

Mycosphere

\section{Comparative evaluation of total peroxidase and catalase activities during light emission of luminous fungus Neonothopanus nambi}

\author{
Mogilnaya OA*, Ronzhin NO and Bondar VS
}

Institute of Biophysics of Siberian Branch of Russian Academy of Sciences, 660036 Krasnoyarsk, Russia

Mogilnaya OA, Ronzhin NO, Bondar VS 2016 - Comparative evaluation of total peroxidase and catalase activities during light emission of luminous fungus Neonothopanus nambi. Mycosphere 7(4), 499-510, Doi 10.5943/mycosphere/7/4/9

\begin{abstract}
Submerged cultivation of luminous fungus Neonothopanus nambi under orbital stirring causes formation of pellets with smooth or rough surfaces. The experiments showed that luminescence of the pellets washed in water increased considerably. Previous studies suggested possible participation of peroxidases in the light emitting reaction. In this study, oxidative azo coupling reaction accompanied by formation of chromogen was used to evaluate peroxidase activity in vivo, in brightly luminescent pellets and in pellets with low luminescence intensity (dim ones). Staining of the brightly luminescent pellets took a few minutes, and their staining intensity was several times higher than that of the dim pellets. From the results of in vivo experiments it was concluded that the bright pellets differed from the dim ones in the production of hydrogen peroxide, or, possibly, other peroxides. Measurements of total peroxidase and catalase activities in pellet extracts also showed an increase in enzyme activities along with an increase in luminescence intensity of native pellets. However, results of the in vitro experiments do not definitively suggest a direct relationship between luminescence and activity of these enzymes. We assume that luminescence of this fungal species may be an additional way to neutralize peroxide compounds under stress.
\end{abstract}

Keywords - basidiomycetes - hydrogen peroxide - luminescence - stress

\section{Introduction}

Isolation and characterization of enzymes catalyzing luminescence reactions (luciferases) and their substrates (luciferins) provided insight into luminescent systems and mechanisms of luminescence of many living organisms (Shimomura 2006). However, molecular organization of the luminescent system of higher fungi and the mechanism of light emitting reaction occurring in them remain poorly understood. Firstly, it is still unclear which enzyme (or enzyme system) functions as luciferase in the fungi and what structure the substrate of luminescence reaction, luciferin, has. In the mid-20th century, Airth and Foerster $(1962,1964)$ showed in their experiments with luminous fungi that luminescence reaction involves two thermolabile protein components soluble NADPH-dependent reductase and luciferase, which are present in the form of insoluble particles - and thermostable luciferin and NADPH. The same result was more recently obtained by other research teams (Kamzolkina et al. 1983, 1984, Oliveira et al. 2009, 2012). To date the study on the molecular mechanism behind the bioluminescence from the mycelium of luminous fungi has 
progressed. Components, which activated the bioluminescence were isolated and chemically characterized (Purtov et al. 2015, Teranishi 2016). However, the structure and properties of luciferase, NADPH-dependent reductase of the fungi remain unknown as these components have not been isolated in pure form yet.

Previously, Shimomura with colleagues (Shimomura 1992, Shimomura et al. 1993) suggested that reactive oxygen species (ROS) and oxidase enzymes played a role in the mechanism of luminescence of higher fungi. A study of six species of luminous fungi showed that ROS, including hydrogen peroxide $\left(\mathrm{H}_{2} \mathrm{O}_{2}\right)$, are needed for strong light emission. Our studies of luminescent fungus Neonothopanus nambi also provided evidence for participation of ROS and oxidase enzymes in the mechanism of fungal luminescence (Bondar et al. 2011-2014, Kobzeva et al. 2014). We found that addition of millimolar concentrations of $\mathrm{H}_{2} \mathrm{O}_{2}$ to mycelium samples (with steady-state or declining luminescence intensities) caused an increase in 3-5-fold in light emission by the fungus. This suggested an assumption that hydrogen peroxide (and perhaps other peroxide compounds) was a necessary component for luminescence reaction of luminous fungus $N$. nambi. In turn, this may indicate that the enzymes with oxidase function (primarily, peroxidases and catalases) could directly or indirectly be involved in the mechanism of fungal luminescence or affect this process.

It is well-known that $\mathrm{H}_{2} \mathrm{O}_{2}$ is among the major metabolites formed during cellular respiration. Higher fungi have various enzymes for utilization of ROS, such as superoxide dismutases, catalases, and peroxidases (Bai et al. 2003, Angelova et al. 2005, Gessler et al. 2007, De Castro et al. 2013, Breitenbach et al. 2015).

There are such peroxidases as secretory, cytosolic, and microsomal ones or peroxidases localized in cell organelles. Most of them are heme-containing enzymes, which carry iron in the active site of protoporphyrin ring. Heme-containing peroxidases of basidiomycetes have been studied and described in sufficient detail as an important factor in the life of fungi and as enzymes with high biotechnological potential (Conesa et al. 2002, Martinez 2002, Kersten \& Cullen 2007, Hofrichter et al. 2010, Ruiz-Duenas \& Martınez 2010, Janusz et al. 2013, Knop et al. 2015). Fungal catalases have been most thoroughly studied in zygomycetes and filamentous ascomycetes (Thieringer et al. 1991, Bourdais et al. 2012, Hansberg et al. 2012). It was shown that these enzymes function intracellularly or may be secreted, demonstrating the highest catalytic activity at high $\mathrm{H}_{2} \mathrm{O}_{2}$ concentrations.

Fungal heme-peroxidases usually localize outside of the cell wall, and they are less effective than catalase in $\mathrm{H}_{2} \mathrm{O}_{2}$ utilization. Moreover, high $\mathrm{H}_{2} \mathrm{O}_{2}$ concentrations (up to $200 \mu \mathrm{M}$ ) may even inactivate extracellular peroxidases (Böckle et al. 1999, Mishra and Imlay 2012, Vidossich et al. 2012). Different compartmentalization of the enzymes (extracellular peroxidases and intracellular catalase) may facilitate effective removal of peroxide compounds.

Taking into account the activating effect of $\mathrm{H}_{2} \mathrm{O}_{2}$ on the light emission of luminous fungus $N$. nambi, we assumed that the peroxidases or peroxide compounds could be involved in this processes. In this study we investigated the total peroxidase and catalase activities in extracts of mycelium of $N$. nambi in comparison with luminescence intensity. Also we examined the activity of extracellular peroxidases in mycelial pellets with and without added horse radish peroxidase (HRP).

\section{Materials \& methods}

\section{Material}

The luminous fungus $N$. nambi (IBSO 2307) is available in the CCIBSO 836 collection of the Institute of Biophysics, Siberian Branch of Russian Academy of Sciences.

\section{Culture medium and procedure}

Submerged cultivation of mycelium was performed in potato sucrose broth (potato broth $200 \mathrm{~g} / \mathrm{L}$, sucrose $20 \mathrm{~g} / \mathrm{L}$ ) in 250-ml conical flasks containing $100 \mathrm{ml}$ of the broth. Mycelium, which 
had been grown in Petri dishes in potato sucrose broth for 8-10 d, was crushed and used as inoculum for submerged cultivation of the fungus. The volume of the inoculum was $2-5 \%(\mathrm{w} / \mathrm{v})$ of the broth volume. Cultivation was carried on for 3-5 d at a temperature of $27-28{ }^{\circ} \mathrm{C}$ under continuous stirring at 180-200 rpm (Environmental Shaker-Incubator ES-20, "BIOSAN", Latvia). By using this procedure, mycelium pellets prepared were 2-7-mm diameter.

\section{Luminescence measurement}

After 3-6 d of cultivation, the broth was removed, and pellets were washed in deionized water (DI water) for several days, with gentle shaking. Luminescence was measured every day in $2.0 \mathrm{ml}$ Eppendorf tubes: 1-3 pellets were placed in $1 \mathrm{ml}$ DI water. Measurements were carried out using a Glomax 20/20 luminometer (Promega) calibrated against Hastings - Weber standard (Hastings \& Weber 1963). One unit is $2.7 \times 10^{3}$ photons in $1 \mathrm{~s}$. After luminescence measurements, the pellets were dried in a rotational vacuum concentrator (Concentrator 5301, Eppendorf, Germany) at $45{ }^{\circ} \mathrm{C}$ for $1 \mathrm{~h}$, for determination of their dry weight. Specific luminescence activity of mycelium was determined as the ratio of the light emission intensity of the sample to its dry weight.

\section{In vivo staining procedure}

Total peroxidase activity was measured, by using the azo coupling reaction. The wellknown azo coupling reaction between 4-aminoantipyrine (4-AAP) and phenol that is catalyzed by peroxidase in the presence of $\mathrm{H}_{2} \mathrm{O}_{2}$ is accompanied by formation of chromogen (quinoneimine). Peroxidase activity of native mycelial pellets was determined in this reaction with some modifications; only phenol $(0.56 \mathrm{mg} / \mathrm{ml})$ and 4-AAP $(0.1 \mathrm{mg} / \mathrm{ml})$ were introduced into the reaction. Ten $\mu \mathrm{l}$ of each reagent and $980 \mu \mathrm{l}$ of DI water were taken per one $\mathrm{ml}$ of the total volume. In the basic experiment, peroxidase and $\mathrm{H}_{2} \mathrm{O}_{2}$ were not added to the reaction. $N$. nambi pellets of the same size were placed in a test tube containing DI water, phenol and 4-AAP. After $1 \mathrm{~h}$ of incubation of pellets at room temperature $\left(25^{\circ} \mathrm{C}\right)$, the dye solution was collected, and absorbance of chromogen was measured in a UV-1800 spectrophotometer (Shimadzu, Japan) at a wavelength of $506 \mathrm{~nm}$.

In a parallel experiment, HRP (1.11.1.7) (Serva) at concentration $0.02 \mathrm{mg} / \mathrm{ml}$ was added to the reaction mixture containing phenol $(0.56 \mathrm{mg} / \mathrm{ml})$ and 4 -AAP $(0.1 \mathrm{mg} / \mathrm{ml})$. The total volume of the reaction mixture was also one $\mathrm{ml}$. Then the pellets were placed into the reaction mixture and incubated for $1 \mathrm{~h}$. Also note that no $\mathrm{H}_{2} \mathrm{O}_{2}$ was introduced into the reaction.

Each measuring experiment was performed in triplicate. Control experiment was nonenzymatic reaction of chromogen formation. In this case, the reaction mixture included phenol, 4AAP, and $\mathrm{H}_{2} \mathrm{O}_{2}$ (without HRP and fungal pellets).

\section{Estimation of binding of phenol and 4-AAP molecules with HRP molecules in a model experiment}

The binding of phenol and 4-AAP molecules with HRP molecules was estimated by adding HRP solution (at a final concentration of $20 \mu \mathrm{g} / \mathrm{ml}$ ) in test-tubes containing phenol solution (14 $\mu \mathrm{g} / \mathrm{ml})$ or a 4 -AAP solution $(5 \mu \mathrm{g} / \mathrm{ml})$. Control test-tubes contained phenol solution or a 4-AAP solution at the same concentrations but without the enzyme. All samples were mixed and incubated for $5 \mathrm{~min}$ at room temperature. The enzyme was separated by dialysis of the samples through the membrane with an exclusion limit of $10 \mathrm{kDa}$ (Amicon ${ }^{\circledR}$ Ultra-0.5, EMD Millipore, Darmstadt, Germany). The dialysis tubes, each containing $500 \mu \mathrm{l}$ of the sample, were centrifuged in a Centrifuge $5415 \mathrm{R}$ for $45 \mathrm{~min}$ at $7,000 \mathrm{~g}$ and $10{ }^{\circ} \mathrm{C}$. Aliquots of $330 \mu \mathrm{l}$ were taken from each submembrane fraction and mixed with $670 \mu \mathrm{l}$ DI water, and then their absorption was analyzed in a UV-1800 spectrophotometer. Aliquots of $330 \mu \mathrm{l}$ were taken from each control tube, mixed with $670 \mu 1 \mathrm{DI}$ water, and subjected to spectral analysis. Binding of phenol and 4-AAP molecules with HRP was estimated from the difference in the absorption spectra of the control and tested samples, by using the values of the optical density of the solutions at a wavelength of $270 \mathrm{~nm}$ for phenol and $243 \mathrm{~nm}$ for 4-AAP. Each measuring experiment was performed in triplicate. 


\section{Measurements of catalase and total peroxidase activities in crude extracts of pellets}

The pellets were washed in distilled water and placed into a glass in an ice bath. A cooled $50 \mathrm{mM}$ Tris- $\mathrm{HCl}$ buffer ( $\mathrm{pH}$ 6.9) was added to the biomass at a ratio of 1:1.5 (wet biomass weight : buffer volume). Biomass was destroyed by sonication at the power $97 \mathrm{~W}$ by using an UZTA 0.63/22-O ultrasonic disintegrator (Volna, Russia). Ultrasonic treatment was repeated 5 times, each lasting 5-7 s, with 1 min intervals.

The resulting biomass was centrifuged at $16,000 \mathrm{~g}$ for $20 \mathrm{~min}$ at a temperature of $4{ }^{\circ} \mathrm{C}$ in Centrifuge $5415 \mathrm{R}$; the supernatant was collected and frozen at $-20{ }^{\circ} \mathrm{C}$. The sediment was dried in a rotational vacuum Concentrator 5301 for $1.5 \mathrm{~h}$ at $30{ }^{\circ} \mathrm{C}$, for determination of dry biomass weight. The frozen supernatants were thawed at room temperature and centrifuged again, under the conditions mentioned above; then, the newly prepared supernatants were collected and used in the measurements of activities of enzymes.

An azo coupling reaction also was used to measure peroxidase activity in supernatants. Supernatant $(980 \mu \mathrm{l})$ was placed in the cuvette and then phenol and 4-AAP (10 $\mu 1$ of each reagent) were added to get the reaction started. The total volume of the reaction mixture was one ml. HRP and $\mathrm{H}_{2} \mathrm{O}_{2}$ were not added to the reaction mixture. The formation of quinoneimine dye was monitored for $60 \mathrm{~min}$ at $506 \mathrm{~nm}$ by a UV-1800 spectrophotometer. One relative unit of peroxidase activity was defined as the chromogen absorbance per unit of total protein in the sample.

Catalase activity in supernatants was measured by the method described in the Catalase assay kit technical bulletin by Sigma-Aldrich Company. Catalase activity was estimated on a UV1800 spectrophotometer by direct UV assay reaction, by monitoring the decrease in absorbance of exogenous $\mathrm{H}_{2} \mathrm{O}_{2}$ added to supernatants for one minute at $240 \mathrm{~nm}$. The total volume of the reaction mixture was one $\mathrm{ml}$. One enzyme unit was defined as the amount of catalase that decomposes $1 \mu \mathrm{M}$ of $\mathrm{H}_{2} \mathrm{O}_{2}$ to $\mathrm{O}_{2}$ and water per minute at $\mathrm{pH} 7.0$ at $25^{\circ} \mathrm{C}$ at a substrate concentration of $10 \mathrm{mM} \mathrm{H}_{2} \mathrm{O}_{2}$.

\section{Determination of total protein in extracts}

Total protein microquantitation in the prepared extracts was performed by biuret method using Benedict's reagent (Kochetov 1980). Samples were incubated for $15 \mathrm{~min}$ at room temperature, and then absorbance was measured using a UV-1800 spectrophotometer at a wavelength of $330 \mathrm{~nm}$.

\section{Visualization of luminescence and images of pellets}

Visual observation of luminescence of native pellets was conducted by using a ChemiDoc $^{\text {TM }}$ XRS System (Bio Rad, USA) in a dark cabinet, in the signal accumulation mode. Exposure time, $300 \mathrm{~s}$, was chosen by trial and error. Images of pellets stained in the azo coupling reaction were made with a PowerShot S50 camera (Canon, Japan). Micrographs of the pellets were obtained by using an AxioImager M2 (Zeiss, Germany) microscope in the transmitted light mode.

\section{Results}

\section{Pellet morphology and luminescence}

By submerged cultivation of $N$. nambi mycelium under orbital stirring, we produced spherical pellets $2-5-\mathrm{mm}$ in diameter, with long or short mycelial outgrowths. They were called smooth and rough pellets (Fig. 1). Surface roughness is caused by bundles of long hyphae extending for distances that might reach a few millimeters. In smooth pellets, the hyphae outgrowths were shorter and they extended for distances of up to $0.5 \mathrm{~mm}$, in the direction perpendicular to the pellet surface.

Mycelial pellets in the liquid nutrient medium emitting $10^{6}-10^{7}$ Quanta $\mathrm{s}^{-1} \mathrm{mg}^{-1}$ were identified as dim. When the pellets were placed in DI water, their luminescence increased by 3 to 5 orders of magnitude (to $10^{9}-10^{12}$ Quanta $\mathrm{s}^{-1} \mathrm{mg}^{-1}$ ). The pellets having increased intensity of luminescence were classified as bright. The mycelium luminescence peak was generally reached in 2-3 d after the fungus was placed in the DI water (Fig. 2). The results showed that large and small pellets could emit bright light (Fig. 3). The surface of small (2-3 mm diam) smooth pellets was, as a rule, uniformly luminescent. Large $(4-5 \mathrm{~mm})$ smooth pellets emitted light either from the entire 
surface or from part of the surface. Small pellets with the bundles of long hyphae showed the brightest luminescence while incubated in water. Large rough pellets, with the bundles of long hyphae, had bright luminescent spots at the base of the bundles.

\section{In vivo staining of pellets for detection of peroxidase activity}

Our results showed that formation of chromogen in azo coupling reaction with native pellets was influenced by their luminescence intensity and the morphology and size of the pellets. The peroxidase reaction that occurred in the pellets is shown in Figure 4. When brightly luminous pellets, both rough and smooth, were placed in the reaction mixture containing phenol and 4-AAP, they were stained pink in 15-20 min. Small brightly luminous pellets (2-3-mm diam) with rough surface having numerous mycelial outgrowths were stained most intensely and rather quickly (after 4-5 min). Dimly luminous pellets of different morphology were stained slowly and weakly for the same time period and under the same conditions. After one hour, the differences in the peroxidase activity staining between bright and dim pellets became more pronounced. In addition, chromogen was not observed in the water at the beginning of the reaction. As the reaction time increased, the chromogen gradually diffused from the pellets to the solution. No staining of the intracellular structures was observed in mycelium samples under a light microscope. Chromogen traces were only seen between the interwoven hyphae.

The results obtained showed distinct differences between brightly luminous and dimly luminous pellets in staining corresponding to peroxidase activity. Therefore in subsequent experiments HRP was added to the reaction mixture containing phenol and 4-AAP, and then the pellets were placed into the Eppendorf tubes. We received an unexpected result, which showed that the brightly luminous pellets were stained to a lesser extent than in the basic experiment (Fig. 5). In the reaction with dimly luminous pellets, no difference was observed in chromogen formation between the experiments with and without HRP.

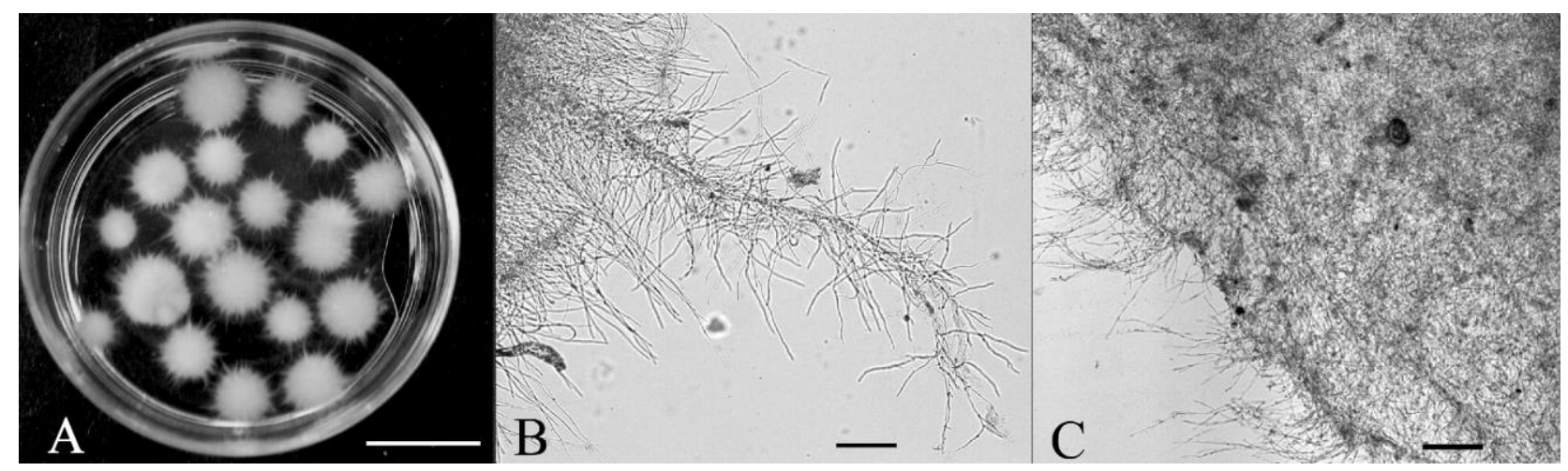

Fig. 1 - Images of fungal pellets grown under submerged cultivation. A Neonothopanus nambi pellets in a Petri dish. B Light micrograph of a rough surface pellet with long hyphal bundles obtained in transmitted light mode. C Light micrograph of a smooth surface pellet with short hyphae. - Bars $=10 \mathrm{~mm}(\mathrm{~A})$ and $100 \mu \mathrm{m}(\mathrm{B}, \mathrm{C})$.

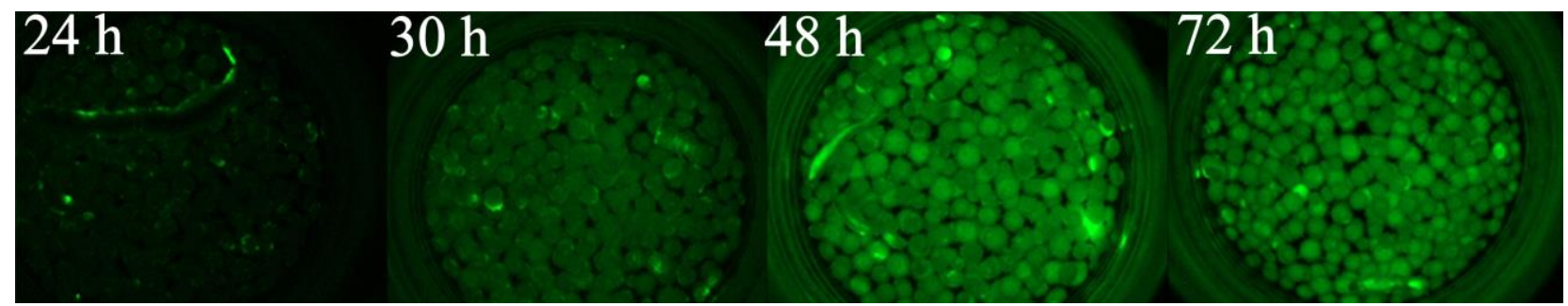

Fig. 2 - Light emission of Neonothopanus nambi pellets incubated in DI water. The images of pellets were performed using Image System (BioRad) in a dark cabinet in the signal accumulation mode. The time exposure was $300 \mathrm{~s}$. The washing periods (h) are indicated in the photographs. 


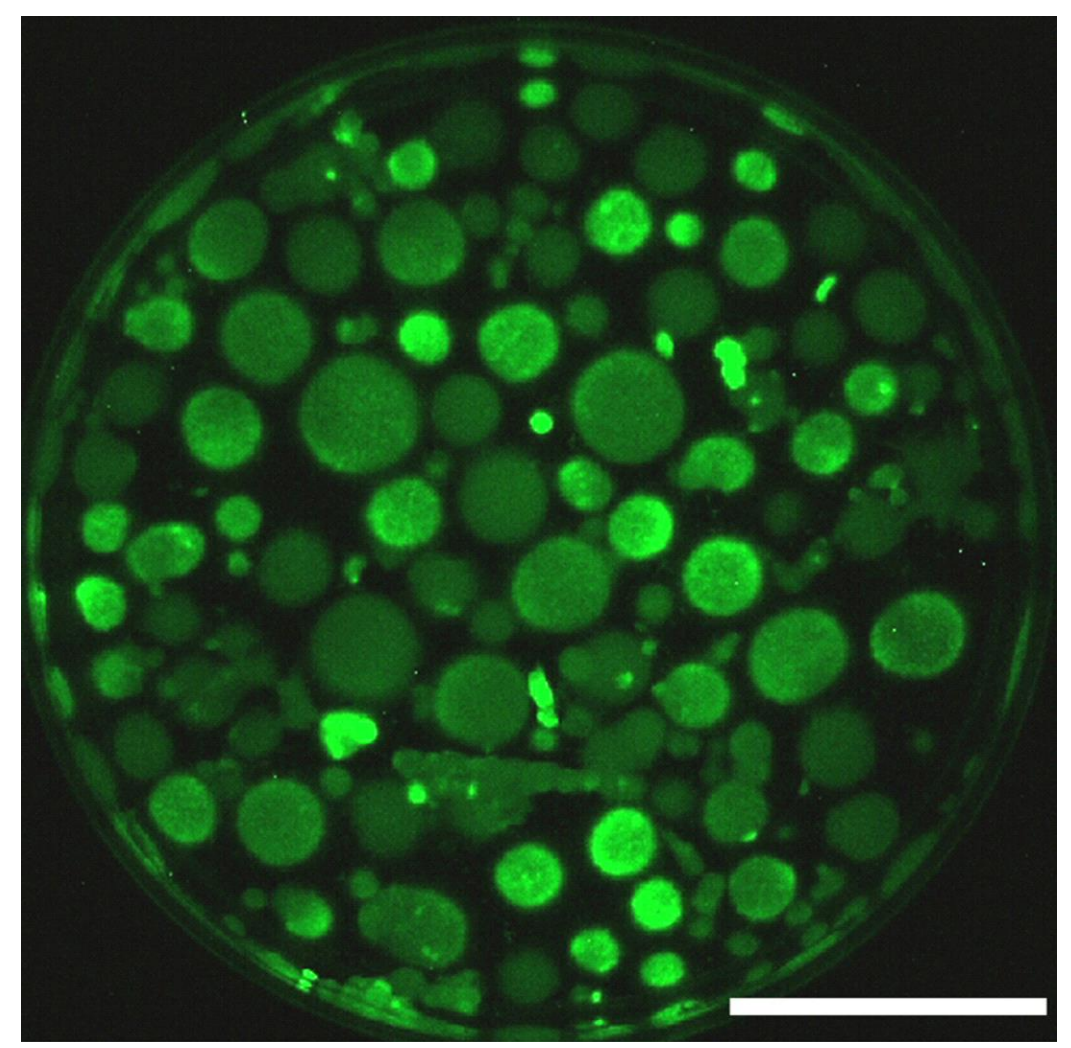

Fig. 3 - Heterogeneity in the distribution of light emission in pellets with different morphological characters. - Bar $=10 \mathrm{~mm}$.

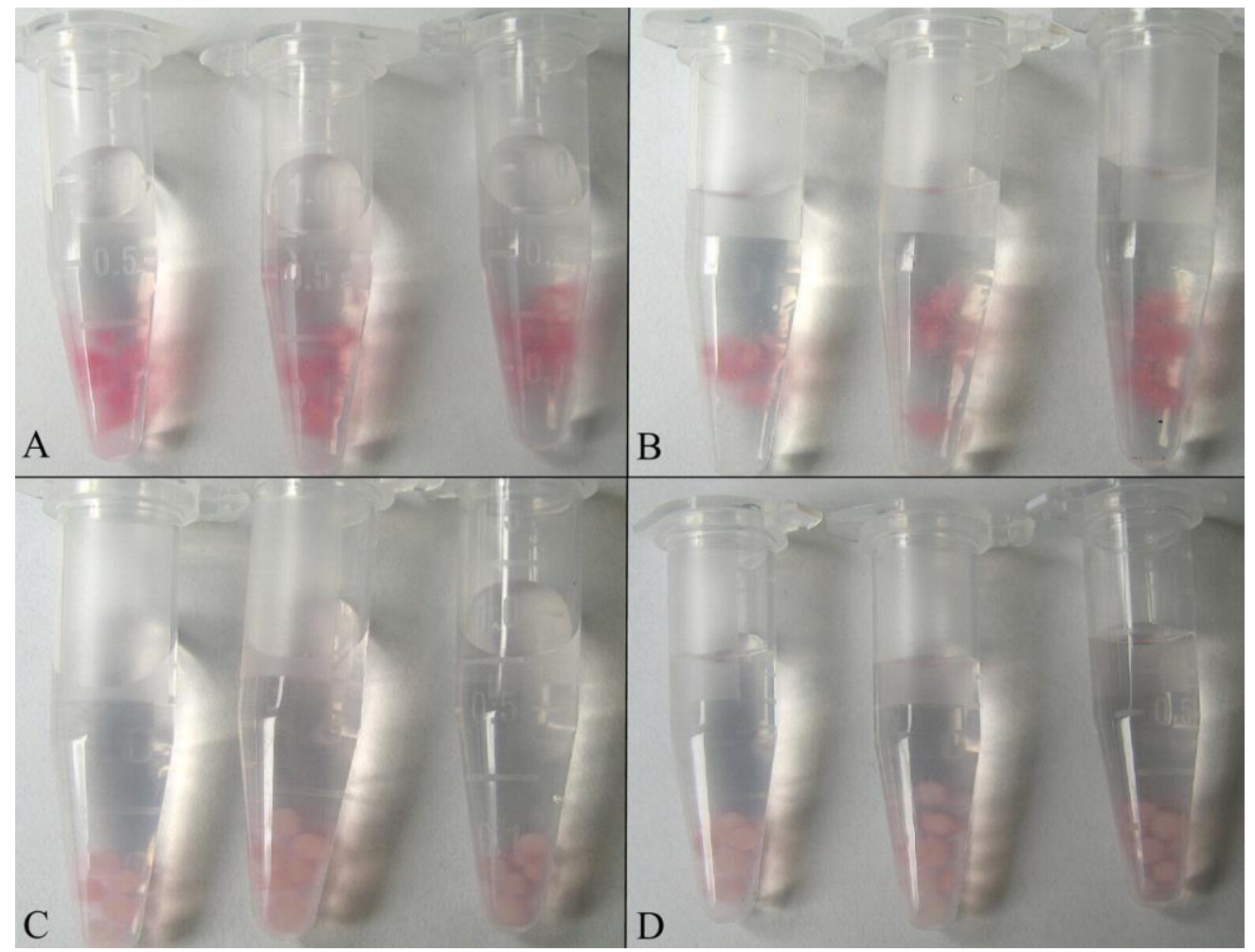

Fig. 4 - Chromogen production in azo coupling reaction with native pellets of Neonothopanus nambi. Images of the stained brightly luminous pellets (A, B) and dimly luminous pellets $(\mathrm{C}, \mathrm{D})$ in 15 min after the reaction initiation. The reactions were carried out in the absence of HRP (A, C) and in the presence of $\operatorname{HRP}(\mathrm{B}, \mathrm{D})$. 


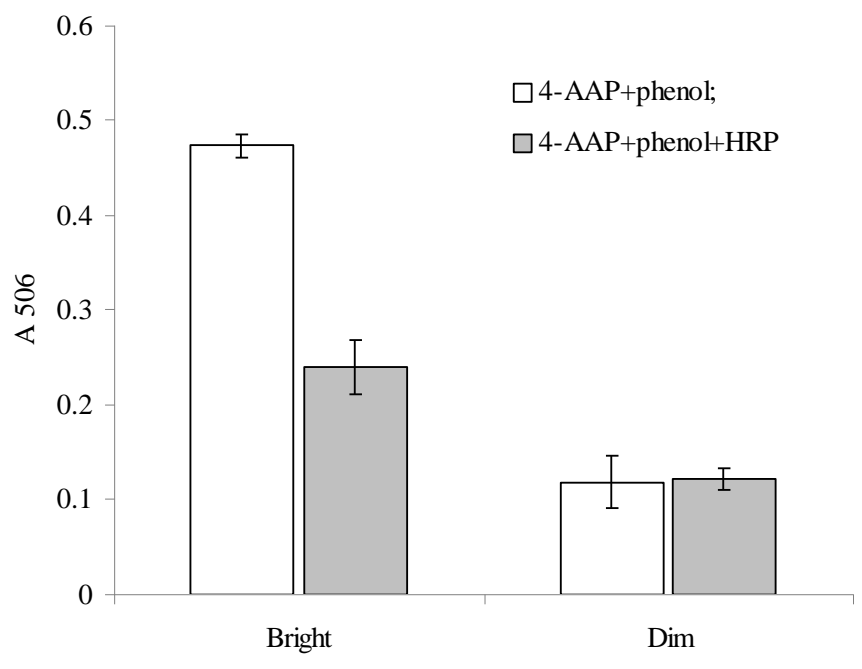

Fig. 5 - The diagram shows the level of chromogen production in the reaction with the brightly luminous and dim pellets in the presence (gray columns) and in the absence of HRP (empty columns).

In model experiments, we tested whether phenol and 4-AAP molecules could bind with added enzyme (HRP) molecules in the absence of hydrogen peroxide. Each of the reagents was incubated with the enzyme, and then enzyme molecules were separated by dialysis. Our results show that under experimental conditions used in this study, the enzyme can bind 4-AAP molecules rather effectively (16\% or more), and phenol molecules are bound by HRP to a lesser extent (about 5\%) (Fig. 6).

\section{Evaluation of catalase and total peroxidase activities in extracts (in vitro)}

To compare the catalase and total peroxidase activities in extracts of brightly and dimly luminous pellets, we also washed the fungus in the DI water for several days. In this way, we were able to monitor changes in pellet luminescence intensity and relate it to the catalase and total peroxidase activities in pellet extracts.
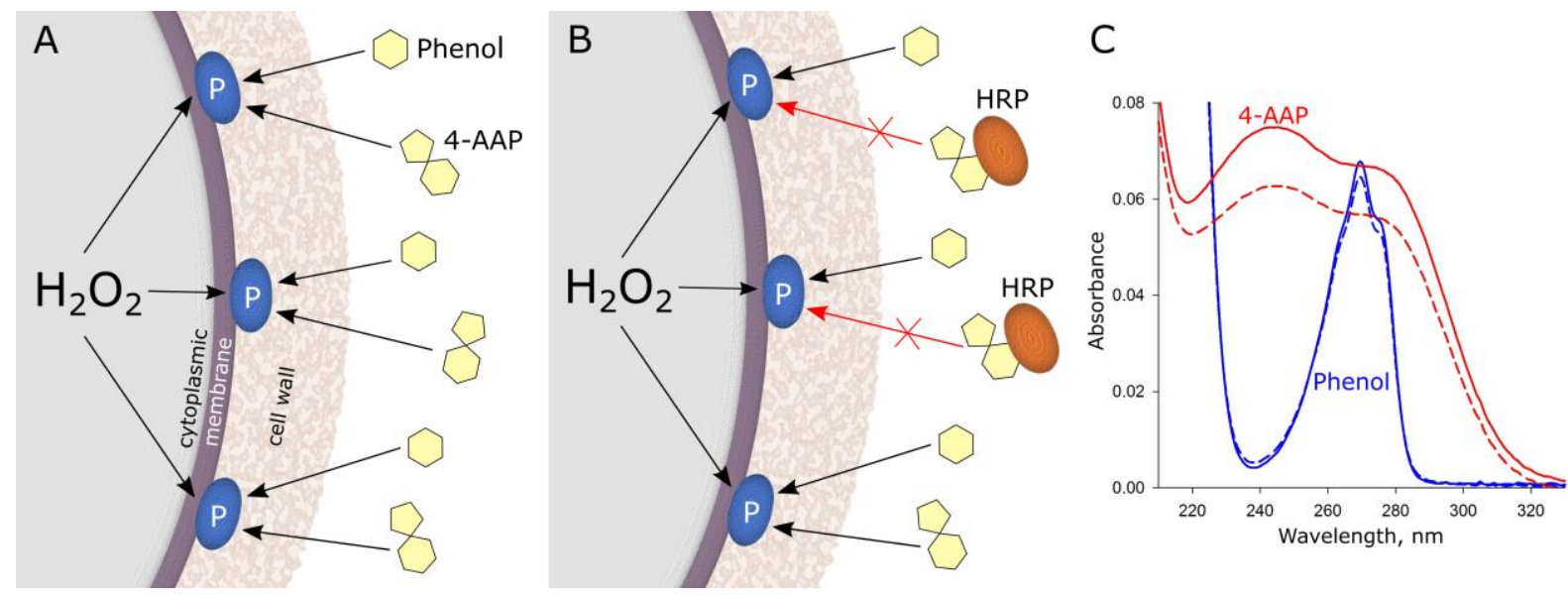

Fig. 6 - A hypothetic scheme of interaction between extracellular peroxidases of the fungus $(\mathrm{P})$ and substrates in an azo coupling reaction. A: In the absence of HRP; B: In the presence of HRP; C: Absorption spectra of 4-AAP and phenol solutions without incubation with HRP (solid lines, control) and after their incubation with HRP and subsequent separation of the enzyme molecules by dialysis (dashed lines). 
As noted above, the pellets collected from the nutrient medium showed low luminescence intensity. During the course of washing, pellet luminescence intensity increased, reached its maximum, and declined. After one week of washing, pellet luminescence decreased considerably, so that it was not recorded with the Image System in the dark cabinet with the exposure time $300 \mathrm{~s}$.

Monitoring of changes in the total peroxidase activity in the extracts of pellet mycelium showed that the activity increased with time, when the pellets were incubated in the DI water (Fig. 7). The lowest activity was observed in the extracts of pellets collected from the nutrient medium (before they were washed in water). The peak of activity was reached after several days of washing and usually later than the peak of luminescence was observed. No peroxidase activity was detected in either culture medium or DI water used to wash the pellets.

Evaluation of the catalase activity in mycelium extracts showed that it increased 10 -fold or even more during the washing of the pellets compared to catalase activity in the extracts of pellets that had not been subjected to stress yet (when they stayed in the culture medium) (Fig. 7). Compared to the peroxidase activity, catalase activity grew quickly, reaching its peak after 1 or $2 \mathrm{~d}$ of incubation in water. No catalase activity was detected in either culture medium or water used to wash the pellets.

\section{Discussion}

Cultivation of $N$. nambi in laboratory experiment showed that pellets prepared in submerged culture under stirring are a convenient form to be used in research. The necessary amounts of materials differing in morphology, size or luminescence intensity can be collected without damaging the mycelium. Previous studies showed that stress conditions such as mechanical damage of fungus $N$. nambi or its incubation in DI water caused a multifold increase in its luminescence intensity (Bondar et al. 2011, 2012, Medvedeva et al. 2014).

In this study, we evaluated the luminescence intensity and total peroxidase activity of $N$. nambi grown in submerged culture and then placed under stress conditions. The results were obtained in native pellets and in their extracts. We proceeded from the assumption that the pellets contained peroxide compounds and peroxidases, therefore, in the basic experiment, phenol as reducing substrate and 4-AAP as coupling reagent for generating quinoneimine dye were only added to the reaction mixture. Additional HRP was used only for comparison in a parallel experiment with the native pellets.

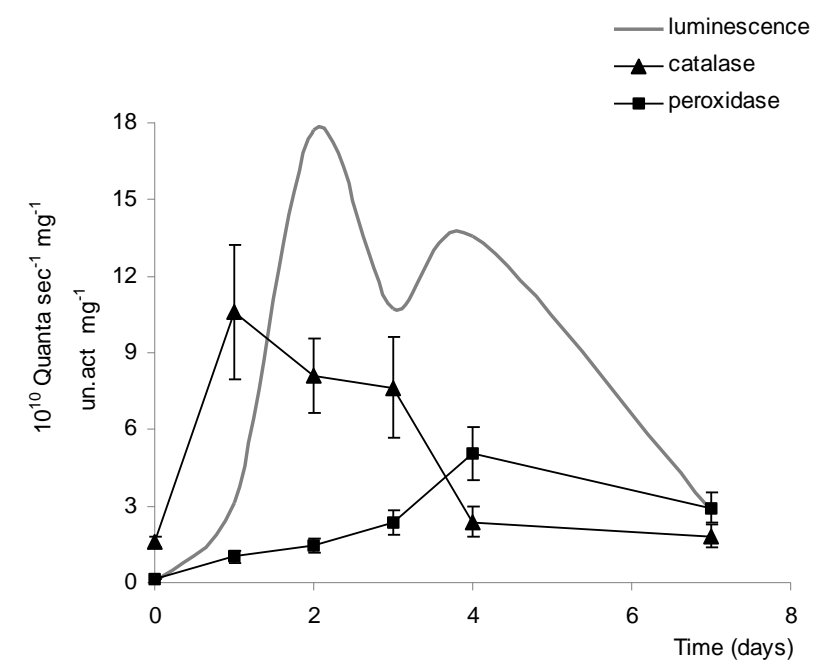

Fig. 7 - Light emission of native Neonothopanus nambi pellets during their incubation in DI water, total peroxidase and catalase activities in extracts of mycelium. $\mathrm{X}$ axis indicates the period when the pellets were washed in DI water. Zero corresponds to the day when the pellets were removed from the culture medium. 
Literature data and our observations suggest that the size and morphology of pellets influence the biosynthetic activity of mycelium. Thus, mass exchange conditions and oxygen availability will differ for peripheral hyphae, especially ones that extend over distances of several $\mathrm{mm}$, and the hyphae situated deep inside the pellet. Leisola et al. (1985) and Ha et al. (2001) reported that the highest activities of lignin peroxidase in Phanerochaete chrysosporium and manganese peroxidase in Pleurotus ostreatus were observed in the culture pellets about $2 \mathrm{~mm}$ diam. In our case, metabolic activity of mycelium of $N$. nambi pellets may be reflected by the heterogeneous distribution of light emission over the pellet surface in the experiment and by the rate and intensity of chromogen formation in the azo coupling reaction with native pellets. As mentioned above, small (2-3-mm diam) pellets showed the brightest luminescence while incubated in water. We can assume that, when placed in DI water, small rough and smooth pellets are more sensitive to stress conditions (such as starvation and osmotic pressure) than larger pellets, which accumulate greater biomass.

In the in vivo staining of $N$. nambi pellets, we showed that brightly luminous pellets significantly differed from dimly luminous pellets in the rate and intensity of the quinoneimine formation. Hence, the fungal pellets differing in the luminescence intensity produced different amounts of extracellular peroxidases or $\mathrm{H}_{2} \mathrm{O}_{2}$. It is known that extracellular fungal peroxidases can be found at a certain distance from the hyphae in the hyphae cell wall and in the slime sheath around the hyphae (Ruel \& Joseleau 1991, Takano et al. 2009). The staining behavior of the $N$. nambi pellets, namely, detection of the chromogen in the intercellular space under microscopic observation, and the gradual diffusion of the chromogen from the pellets to the solution also indicate that the azo coupling reaction occurred outside of the hyphae, where extracellular peroxidases of the fungus are localized.

In the parallel in vivo experiment, with HRP added to the reaction mixture, we observed a decrease in the intensity of chromogen formation by the brightly luminous pellets. We suppose that this effect may be caused by partial spatial separation between extracellular peroxidases of the fungus and phenol and 4-AAP and between HRP and $\mathrm{H}_{2} \mathrm{O}_{2}$. As we mentioned in the Materials and Methods section, in the experiment with native pellets, we first prepared the reaction mixture and only then placed pellets into it. The enzyme HRP present in the solution could probably bind part of the phenol and 4-AAP, precluding their interaction with fungal extracellular peroxidases. The in vitro model experiment showed that HRP molecules bound 4-AAP molecules (16\% or more) and, to a lesser extent, phenol molecules in the absence of $\mathrm{H}_{2} \mathrm{O}_{2}$. Thus, before pellets were placed in the reaction mixture, part of 4-AAP and phenol molecules could be bound to the HRP and be unavailable for fungus peroxidases and unable to take part in the reaction catalyzed by them. In addition to that, when pellets were placed in the reaction mixture containing HRP, pellet mycelium rather than the solution surrounding the pellets was stained first. Thus, the first substrate $\left(\mathrm{H}_{2} \mathrm{O}_{2}\right)$ generated inside the cells and diffused to cell wall had been bound with fungal extracellular peroxidases and, hence, was unavailable for added HRP present in solution.

Thus, both fungal extracellular peroxidases and additional enzyme, HRP, will be deficient in reaction substrates. We think that in this case, the staining of native pellets was affected by different spatial localization of these enzymes and substrates in accordance with the staining procedure described. At least, this is a plausible explanation for the observation times used in this study.

The dimly luminescent fungus pellets showed similar rates of chromogen formation with and without exogenous HRP, which was indicative of the lack of $\mathrm{H}_{2} \mathrm{O}_{2}$ as a substrate. Therefore, irrespective of the presence of HRP in the solution, chromogen formation is limited only by $\mathrm{H}_{2} \mathrm{O}_{2}$ concentration. The above reasoning leads to a more definite conclusion, suggesting that bright pellets differed from dim ones in the production of $\mathrm{H}_{2} \mathrm{O}_{2}$ or other peroxides.

As luminescence of the native pellets washed in water increased dramatically, we studied changes in the total peroxidase and catalase activities in the pellet extracts that occurred during the washing process. The results of in vitro experiments showed that during the course of washing of native pellets, the catalase and total peroxidase activities increased considerably in the extracts of these pellets, too, compared to their levels in the pellets kept in the culture medium. Catalase 
activity in the extracts rose quickly and approached its steady state before the luminescence reached its peak, while the total peroxidase activity increased slowly, reaching its peak with the luminescence peak or even later. It is well known that ROS are continuously produced in the cell, but their normal levels are not high, and the cell inactivates them with the antioxidant system. All living organisms (bacteria, plants, animals) respond to the increased levels of intracellular $\mathrm{H}_{2} \mathrm{O}_{2}$ or the inflow of extracellular $\mathrm{H}_{2} \mathrm{O}_{2}$ by enhanced synthesis of peroxidases and catalases (Bai et al. 2003; Gessler et al. 2007). We observed higher activity of these enzymes in the extracts of pellets incubated for long time periods in DI water, i.e. when the cells were starved and the rates of intracellular oxidative processes increased. There was significant difference in activity profiles between catalase and peroxidase (Fig. 7). Catalase is known to operate through a disproportionation cycle at higher doses of $\mathrm{H}_{2} \mathrm{O}_{2}$, whereas peroxidases reduce $\mathrm{H}_{2} \mathrm{O}_{2}$ via a serial two-step one-electron oxidation of different substrates and typically have a slow reductive stage. They work at low concentrations of $\mathrm{H}_{2} \mathrm{O}_{2}$ (Mishra \& Imlay 2012). From our results we can assume that in the first 1$3 \mathrm{~d}$ of incubation of pellets in DI water, the high level of $\mathrm{H}_{2} \mathrm{O}_{2}$ was neutralized by catalase and also in the luminescence reaction. Therefore, total peroxidase activity increased more slowly, possibly, due to deficiency of $\mathrm{H}_{2} \mathrm{O}_{2}$ or other reductants in the beginning of starvation period. However, results of the in vitro experiments do not definitively suggest a direct relationship between luminescence intensity and activity of these enzymes. We can only conclude that the luminescence of $N$. nambi and activity of these enzymes may be related by the same metabolic basis. At the same time, the fact that the changes in these parameters were unidirectional supports the hypothesis that the light emission of this species of fungus originated during the course of evolution as an additional way to neutralize ROS.

\section{Acknowledgments}

We are grateful to Emma Rodicheva for the cultivation of $N$. nambi. This work was supported by the state budget allocated to the fundamental research at the Russian Academy of Sciences (project no. 0360-2014-0006) and Program No.II.2 «Integration and Development» of the Siberian Branch of the Russian Academy of Sciences (project no. 0360-2015-0003).

\section{References}

Airth RL, Foerster GE. 1962 - The isolation of catalytic components required for cell-free fungal bioluminescence. Archives of Biochemistry and Biophysics 97, 567-573.

Airth RL, Forester GE. 1964 - Enzymes associated with bioluminescence of Panus stipticus luminescens and Panus stipticus non-luminescens. Journal of Bacteriology 88, 1372-1379.

Angelova MB, Pashova SB, Spasova BK, Vassilev SV, Slokoska LS. 2005 - Oxidative stress response of filamentous fungi induced by hydrogen peroxide and paraquat. Mycological Research 109, 150-158.

Bai Z, Harvey LM, McNeil B. 2003 - Oxidative stress in submerged cultures of fungi. Critical Reviews in Biotechnology 23, 267-302.

Böckle B, Martínez MJ, Guillén F, Martínez AT. 1999 - Mechanism of peroxidase inactivation in liquid cultures of the ligninolytic fungus Pleurotus pulmonarius. Applied and Environmental Microbiology 65, 923-928.

Bondar VS, Puzyr AP, Purtov KV, Medvedeva SE, Rodicheva EK, Gitelson JI. 2011 - The luminescent system of the luminous fungus Neonothopanus nambi. Doklady Biochemistry and Biophysics 438, 138-140.

Bondar VS, Puzyr AP, Purtov KV, Petunin AI, Burov AE, Rodicheva EK, Medvedeva SE, Shpak BA, Tyaglik AB, Shimomura O, Gitelson JI. 2014 - Isolation of luminescence system from the luminescent fungus Neonothopanus nambi. Doklady Biochemistry and Biophysics 455, $56-58$. 
Bondar VS, Rodicheva EK, Medvedeva SE, Tyulkova NA, Tyaglik AB, Shpak BA, Gitelson JI. 2013 - On the mechanism of luminescence of the fungus Neonothopanus nambi. Doklady Biochemistry and Biophysics 449, 80-83.

Bondar VS, Shimomura O, Gitelson JI, 2012 - Luminescence of higher mushrooms. Journal of Siberian Federal University. Biology 4, 331-351.

Bourdais A, Bidard F, Zickler D, Berteaux-Lecellier V, Silar P, Espagne E. 2012 - Wood utilization is dependent on catalase activities in the filamentous fungus Podospora anserine. PLoS ONE 7, e29820.

Breitenbach M, Weber M, Rinnerthaler M, Karl T, Breitenbach-Koller L. 2015 - Oxidative stress in fungi: its function in signal transduction, interaction with plant hosts, and lignocellulose degradation. Biomolecules 5, 318-342.

Conesa A, Punt PJ, van den Hondel CA. 2002 - Fungal peroxidases: molecular aspects and applications. Journal of Biotechnology 93, 143-158.

De Castro C, Del Valle P, Rua J, Garsia-Armesto MR, Gutierrez-Larrainzar M, Busto F, De Arriaga D. 2013 - Antioxidant defence system during exponential and stationary growth phases of Phycomyces blakesleeanus: Response to oxidative stress by hydrogen peroxide. Fungal Biology 117, 275-287.

Gessler NN, Aver'yanov AA, Belozerskaya TA. 2007 - Reactive oxygen species in regulation of fungal development. Biochemistry (Moscow) 72, 1091-1109.

Ha H-C, Honda Y, Watanabe T, Kuwahara M. 2001 - Production of manganese peroxidase by pellet culture of the lignin-degrading basidiomycete, Pleurotus ostreatus. Applied and Environmental Microbiology 55, 704-711.

Hansberg W, Salas-Lizana R, Domínguez L. 2012 - Fungal catalases: function, phylogenetic origin and structure. Archives of Biochemistry and Biophysics 525, 170-180.

Hastings JW, Weber G. 1963 - Total quantum flux of isotopic sources. Journal of the Optical Society of America 53, 1410-1415.

Hofrichter M, Ullrich R, Pecyna MJ, Liers C, Lunde T. 2010 - New and classic families of secreted fungal heme peroxidases. Applied Microbiology and Biotechnology 87, 871-897.

Janusz G, Kucharzykb KH, Pawlika A, Staszczaka M, Paszczynski AJ. 2013 - Fungal laccase, manganese peroxidase and lignin peroxidase: gene expression and regulation. Enzyme Microbiology and Technology 52, 1-12.

Kamzolkina OV, Bekker ZE, Egorov NS. 1984 - Extraction of the luciferin-luciferase system from the fungus Armillariella mellea (in Russian). Biologicheskie Nauki 1, 73-77.

Kamzolkina OV, Danilov VS, Egorov NS. 1983 - Nature of luciferase from the bioluminescent fungus Armillariella mellea (in Russian). Doklady Akademii Nauk SSSR 271, 750-752.

Kersten F, Cullen D. 2007 - Extracellular oxidative systems of the lignin-degrading basidiomycete Phanerochaete chrysosporium. Fungal Genetics and Biology 44, 77-87.

Knop D, Yarden O, Hadar Y. 2015 - The ligninolytic peroxidases in the genus Pleurotus: divergence in activities, expression, and potential applications. Applied Microbiology and Biotechnology 99, 1025-1038.

Kobzeva T, Melnikov A, Karogodina T, Zikirin S, Stass D, Molin Y, Rodicheva E, Medvedeva S, Puzyr A, Burov A, Bondar V, Gitelson JI. 2014 - Stimulation of luminescence of mycelium of luminous fungus Neonothopanus nambi by ionizing radiation. Luminescence 29, 703710 .

Kochetov GA. 1980 - Practical guide to enzymology (in Russian). Higher School, Moscow.

Leisola MSA, Thanei-Wyss U, Fiechter A. 1985 - Strategies for production of high ligninase activities by Phanerochaete chrysosporium. Journal of Biotechnology 3, 97-107.

Martinez AT. 2002 - Molecular biology and structure-function of lignin-degrading heme peroxidases. Enzyme and Microbial Technology 30, 425-444.

Medvedeva SE, Artemenko KS, Krivosheenko AA, Rusinova AG, Rodicheva EK, Puzyr AP, Bondar VS. 2014 - Growth and light emission of luminous basidiomycetes cultivated on solid media and in submerged culture. Mycosphere 5, 565-577. 
Mishra S, Imlay J. 2012 - Why do bacteria use so many enzymes to scavenge hydrogen peroxide? Archives of Biochemistry and Biophysics 525, 145-160.

Oliveira AG, Desjardin DE, Perry BA, Stevani CV. 2012 - Evidence that a single bioluminescent system is shared by all known bioluminescent fungal lineages. Photochemical and Photobiological Sciences 11, 848-852.

Oliveira AG, Stevani CV. 2009 - The enzymatic nature of fungal bioluminescence. Photochemical and Photobiological Sciences 8, 1416-1421.

Purtov KV, Petushkov VN, Baranov MS, Mineev KS, Rodionova NS, Kaskova ZM, Tsarkova AS, Petunin AI, Bondar VS, Rodicheva EK, Medvedeva SE, Oba Y, Oba Y, Arseniev AS, Lukyanov S, Gitelson JI, Yampolsky IV. 2015 - The chemical basis of fungal bioluminescence. Angewandte Communications 54, 8124-8128.

Ruel K, Joseleau J-P. 1991 - Involvement of an extracellular glucan sheath during degradation of Populus wood by Phanerochaete chrysosporium. Applird and Environmental Microbiology 57, 374-384.

Ruiz-Duenas FJ, Martınez AT. 2010 - Structural and functional features of peroxidases with a potential as industrial biocatalysts. In: Torres E, Ayala M (eds), Biocatalysis based on heme peroxidases. Springer-Verlag, Berlin.

Shimomura O, Satoh S, Kishi Y. 1993 - Structure and non-enzymatic light emission of two luciferin precursors isolated from luminous mushroom Panellus stipticus. Journal of Bioluminescence and Chemiluminescence 8, 201.

Shimomura O. 1992 - The role of superoxide dismutase in regulating the light emission of luminescent fungi. Journal of Experimental Botany 43, 1519-1525.

Shimomura O. 2006 - Bioluminescence: chemical principles and methods. World Scientific Publishing, Singapore.

Takano M, Hayashi N, Nakamura M, Yamaguchi M. 2009 - Extracellular peroxidase reaction at hyphal tips of white-rot fungus Phanerochaete crassa WD1694 and in fungal slime. Journal of Wood Science 55, 302-307.

Teranishi K. 2016 - trans-p-Hydroxycinnamic acid as a bioluminescence-activating component in the pileus of the luminous fungus Mycena chlorophos. Tetrahedron 72, 726-733.

Thieringer R, Shio H, Han Y, Cohen G, Lazarow PB. 1991 - Peroxisomes in Saccharomyces cerevisiae: immunofluorescence analysis and import of catalase A into isolated peroxisomes. Molecular and Cellular Biology 11, 510-522.

Vidossich P, Alfonso-Prieto M, Rovira C. 2012 - Catalases versus peroxidases: DFT investigation of $\mathrm{H}_{2} \mathrm{O}_{2}$ oxidation in models systems and implications for heme protein engineering. Journal of Inorganic Biochemistry 117, 292-297. 